\title{
Ramos colaterais parietais e terminais da aorta abdominal em Myocastor coypus (nutria)
}

\author{
Terminal and parietal colateral branches of the abdominal aorta in Myocastor coypus (nutria)
}

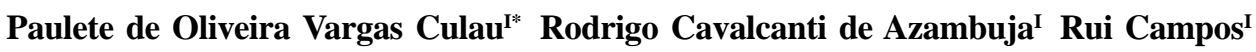

\section{RESUMO}

Neste estudo, utilizaram-se 30 nutrias, 15 fêmeas e 15 machos, com o sistema arterial aórtico-abdominal preenchido com látex 603, pigmentado em vermelho, e fixado em uma solução aquosa de formaldeído a 20\%. A aorta abdominal emitiu de sua superfície dorsal de 6 a 8 artérias lombares únicas. Das artérias renais, direita e esquerda, originaram-se as artérias frênico-abdominal para irrigar parte do diafragma e da parede abdominal lateral cranial. A aorta abdominal lançou dorsalmente, a artéria sacral mediana, cranialmente a sua bifurcação em artérias ilíacas comuns. As artérias ilíacas comuns, ramos terminais da aorta abdominal, originaram as artérias ilíacas interna e externa. A artéria ilíaca interna distribuiu-se nas vísceras da cavidade pélvica. A artéria ilíaca externa emitiu uma artéria umbilical e, antes de alcançar o anel femoral, lançou a artéria circunflexa ilíaca profunda para a parede abdominal lateral, em seus dois terços caudais. A artéria ilíaca externa lançou o tronco pudendo-epigástrico, que originou a artéria epigástrica caudal, para a parede abdominal ventral e a artéria pudenda externa, que saiu pelo canal inguinal, para irrigar a genitália externa. Os ramos parietais diretos da aorta abdominal foram as artérias lombares e a artéria sacral mediana, enquanto as artérias frênicoabdominal, circunflexa ilíaca profunda e epigástrica caudal, foram ramos colaterais parietais indiretos. Os ramos terminais da artéria aorta abdominal foram as artérias ilíacas comuns com seus ramos, as artérias ilíacas interna e externa.

Palavras-chave: artérias abdominais, vascularização, roedor, ABSTRACT nutria, Myocastor coypus.

For this study it was used 30 nutria, 15 females and 15 males, with its abdominal aorta system full filled with latex 603, stained in red, and fixed in an aqueous solution of formaldehyde 20\%. The abdominal aorta emitted from its dorsal surface 6 to 8 single lumbar arteries. From the renal arteries, left and right, it has been originated the phrenicoabdominal arteries, in order to irrigate part of the diaphragm and the cranial lateral abdominal walls. The abdominal aorta emitted, dorsally, the median sacral artery, cranially to its bifurcation into the common iliac arteries. These common iliac arteries, terminal branches of the aorta, originate the internal and external iliac arteries. The internal iliac artery was distributed along the pelvic cavity viscera. The external iliac artery emitted an umbilical artery and, before reaching the inguinal ring, emitted the deep circunflex iliac artery to 2/3 of the caudal lateral abdominal wall. The external iliac artery emitted the pudendoepigastric trunk, wich has originated the caudal epigastric artery, to the ventral abdominal wall, and the external pudendal artery, wich passed through the inguinal ring to irrigate the external genital. The direct parietal branches of the abdominal aorta were the lumbar arteries and the median sacral artery, while the phrenicoabdominal arteries, deep circunflex iliac and the caudal epigastric artery were indirect colateral parietal branches. The terminal branches of the abdominal aorta were the common iliac arteries with its branches, the internal and external iliac arteries.

Key words: abdominal arteries, vascularization, rodents, nutria, Myocastor coypus.

\section{INTRODUÇÃO}

Os roedores (ordem Rodentia) representam o mais numeroso grupo dentro da classe Mammalia. Da família Capromyidae, registra-se no Brasil a ocorrência apenas do gênero Myocastor, com uma única espécie vivente, o Myocastor coypus, conhecido como ratão-do-banhado ou nutria (SILVA, 1994). Tratase de um roedor de tamanho médio, nativo do extremo sul da América do Sul, mas disseminado em várias partes do mundo, como Estados Unidos e Europa, onde tem sido explorado com fins comerciais, em

IPrograma de Pós-graduação em Ciências Veterinárias, Departamento de Ciências Morfológicas, Instituto de Ciências Básicas da Saúde, Faculdade de Veterinária, Universidade Federal do Rio Grande do Sul (UFRGS). Av. Bento Gonçalves, 9090, 91540-000, Porto Alegre, RS, Brasil. E-mail: paulete.culau@ufrgs.br. *Autor para correspondência. 
especial pela qualidade de sua pele e de sua carne (BAROFFIO et al., 1979).

Informações sobre a morfologia do ratãodo-banhado são escassamente encontradas na literatura, salvo abordagens gerias sobre sua pelagem, dentição e volume corporal (SILVA, 1994). Com relação ao suprimento vascular da região abdominal, encontram-se alguns relatos sobre o suprimento arterial para as glândulas adrenais (MACHADO et al., 2002) e sobre a artéria celíaca e seus ramos em ratão do banhado (MACHADO et al., 2002). Assim, para confrontar os resultados, na discussão, utilizamos informações sobre a aorta abdominal em ratos, gambás e cães.

Desse modo, este trabalho busca obter informações de cunho morfológico que possam fundamentar discussões do ponto de vista funcional e resultaem em subsídios para a melhor compreensão da fisiologia desses animais. Assim é possível oferecer suporte para medidas que visem a protegê-los em seu ambiente natural, bem como aprimorar métodos de sua exploração racional. Portanto, o presente trabalho tem como objetivo sistematizar e descrever os ramos parietais e terminais da aorta abdominal em Myocastor coypus.

\section{MATERIAL E MÉTODOS}

Para este estudo, utilizaram-se 30 nutrias (15 fêmeas e 15 machos), jovens e adultas, provenientes de um criatório localizado na cidade de Caxias do Sul, licenciado pelo Instituto Brasileiro de Meio Ambiente e Recursos Naturais Renováveis (IBAMA). Administrou-se aos animais 5000UI de heparina ${ }^{\mathrm{a}}$ e trinta minutos depois $20 \mathrm{ml}$ de Tiopental sódico ${ }^{\mathrm{b}}$ a $12,5 \%$, via intraperitoneal. A caixa torácica foi aberta ventralmente em plastrão, e a aorta torácica foi canulada próximo ao diafragma. O sistema foi lavado com $250 \mathrm{ml}$ de solução salina. Após, preencheu-se o sistema com látex 603, corado em vermelho ${ }^{\mathrm{d}}$ (BASF), e o animal permaneceu em água corrente por uma hora para solidificação do mesmo. Rebateu-se a pele e injetou-se intraperitoneal $150 \mathrm{ml}$ de formaldeído a $20 \%$ e o animal foi imerso nesta solução por sete dias.

Abriu-se a cavidade abdominal na linha mediana ventral e as artérias foram dissecadas. Depois foram confeccionados desenhos esquemáticos de todas as peças e algumas amostras para documentação. As designações seguiram a Nômina Anatômica Veterinária (INTERNATIONAL COMMITTEE ON VETERINARY GROSS ANATOMICAL NOMENCLATURE, 2005).

\section{RESULTADOS}

A aorta atravessou o hiato aórtico entre os pilares do diafragma e, ao emergir na cavidade abdominal, emitiu em seqüência a artéria celíaca e a artéria mesentérica cranial, ventralmente e justapostas.

De sua superfície dorsal, lançou em seqüência e com espaçamento constante de 6 a 8 artérias lombares únicas, que se distribuíram na região lombar, sendo que a última artéria lombar foi quase sempre originada da artéria sacral mediana (Figura 1).

A alguns centímetros, caudalmente à origem da artéria mesentérica cranial, a aorta abdominal emitiu, lateralmente, as artérias renal direita e esquerda. A artéria frênico-abdominal, o primeiro ramo parietal, foi normalmente lançada como ramo colateral das artérias renais (Figuras 1 e 2). Este tronco lançou a artéria frênica caudal, a artéria abdominal cranial e as artérias adrenais em $66,7 \%$ dos casos à direita e em 63,3\% à esquerda. As artérias frênicas caudais, direita e esquerda, dirigiram-se cranialmente para os respectivos pilares do diafragma, irrigando suas porções lombares. A artéria abdominal cranial originou-se do tronco frênicoabdominal caudo-lateralmente, alcançando o terço mais cranial da parede dorsolateral da cavidade abdominal. As várias artérias adrenais, direita e esquerda, foram emitidas lateralmente, cooperando na irrigação da glândula adrenal.

A artéria frênica caudal direita originou-se da artéria frênico-abdominal direita, que era ramo da artéria renal direita em 66,7\% dos casos. Ela surgiu também como ramo colateral da artéria renal direita juntamente com a artéria adrenal em 26,7\% dos achados ou então ela foi ramo colateral da aorta abdominal como um tronco comum com a artéria abdominal cranial e adrenal em 3,3\% dos casos e em 3,3\% das amostras ela era um ramo colateral da artéria celíaca.

A artéria frênica caudal esquerda originouse da artéria frênico-abdominal esquerda, que era ramo da artéria renal esquerda em $63,3 \%$ dos casos. ela também surgiu como ramo da artéria frênico-abdominal esquerda, que era ramo direto da aorta abdominal em $16,7 \%$ dos achados, ou era ramo colateral da artéria renal direita juntamente com a artéria adrenal em 16,7\%, dos casos. Ela também foi ramo colateral da aorta abdominal em 3,3\% das amostras.

A porção lombar do diafragma esporadicamente recebeu uma complementação vascular de uma artéria frênica caudal acessória oriunda de uma fonte alternativa, ou seja, em uma peça à direita, a artéria frênica caudal acessória foi ramo da artéria gástrica esquerda e ramo da artéria celíaca em outra. Já à esquerda, em três dos achados, ela era ramo da artéria gástrica esquerda.

A artéria abdominal cranial direita foi ramo da artéria frênico-abdominal, que foi emitida da artéria renal direita em $63,4 \%$ das amostras. Ela também foi 


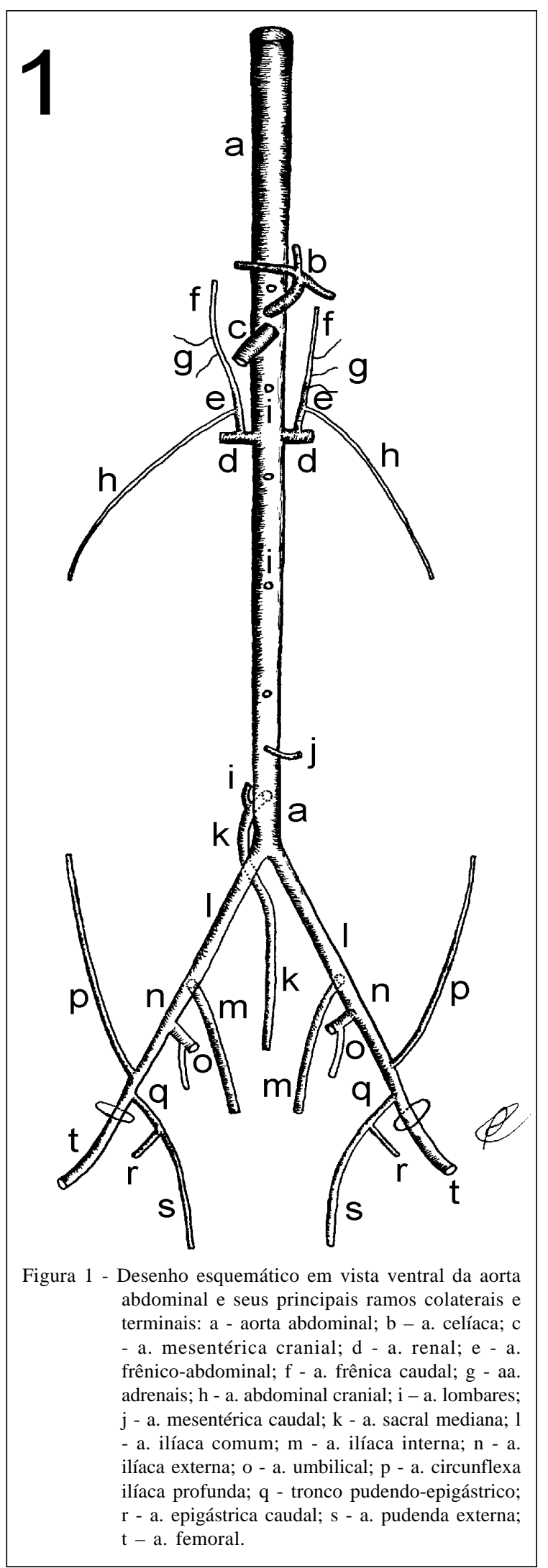

ramo de uma artéria lombar originada da aorta abdominal, caudalmente à origem da artéria mesentérica cranial em $20 \%$ das peças, enquanto foi lançada diretamente da artéria renal direita em $10 \%$ dos casos, ou da artéria frênico-abdominal direita que foi emitida da aorta abdominal em 3,3\% e ainda foi ramo da artéria renal direita juntamente com a artéria adrenal em 3,3\% das amostras.

A artéria abdominal cranial esquerda, ramo da artéria frênico-abdominal, originou-se da artéria renal esquerda em $63,3 \%$ das amostras, enquanto foi ramo da artéria frênico-abdominal esquerda que foi emitida diretamente da aorta abdominal em 16,7\% das peças, ou foi ramo de uma artéria lombar originada da aorta abdominal, caudalmente à origem da artéria mesentérica cranial em 13,3\% dos achados, e ainda foi lançada diretamente da artéria renal esquerda em 6,7\% dos casos.

A artéria sacral mediana, diferentemente dos animais domésticos, originou-se da aorta abdominal dorsalmente em 100\% dos animais (Figura 1), projetando-se profundamente para a cauda, logo após emitir em $83,3 \%$ das peças a última artéria lombar. Em 53,3\% dos casos, a artéria sacral mediana foi lançada pouco depois da emissão ventral da artéria mesentérica caudal. Já em 26,7\% dos achados, a artéria sacral mediana originou-se da aorta abdominal cranialmente à origem da artéria mesentérica caudal, enquanto em $16,7 \%$ das peças ela foi emitida na mesma altura da origem da artéria mesentérica caudal e em 3,3\% não houve ponto de referência devido à ausência da artéria mesentérica caudal.

Próximo à entrada da cavidade pélvica dorsalmente, a aorta abdominal, em todas as preparações, dividiu-se em seus ramos terminais, as artérias ilíacas comuns, direita e esquerda, projetadas caudalmente em afastamento divergente de $30^{\circ}$ a $40^{\circ}$. A artéria ilíaca comum dividiu-se em artéria ilíaca interna, continuando-se em artéria ilíaca externa, depois de um trajeto de dois a três centímetros, em todas as preparações, nos dois antímeros (Figuras 1 e 2).

A artéria ilíaca interna dirigiu-se caudomedialmente, no interior da cavidade pélvica, distribuindo-se nas vísceras da região, por meio de suas ramificações. A artéria ilíaca externa projetou-se caudo-lateralmente em direção ao anel femoral, emitindo junto ou próximo a sua origem a artéria umbilical, em $86,7 \%$ das peças à direita, e $90 \%$ à esquerda, enquanto que em $13,3 \%$ dos casos à direita e em $6,7 \%$ à esquerda, a artéria umbilical foi ramo colateral da artéria ilíaca comum e em 3,3\% à esquerda foi ramo colateral da ilíaca interna.

Ciência Rural, v.38, n.4, jul, 2008. 


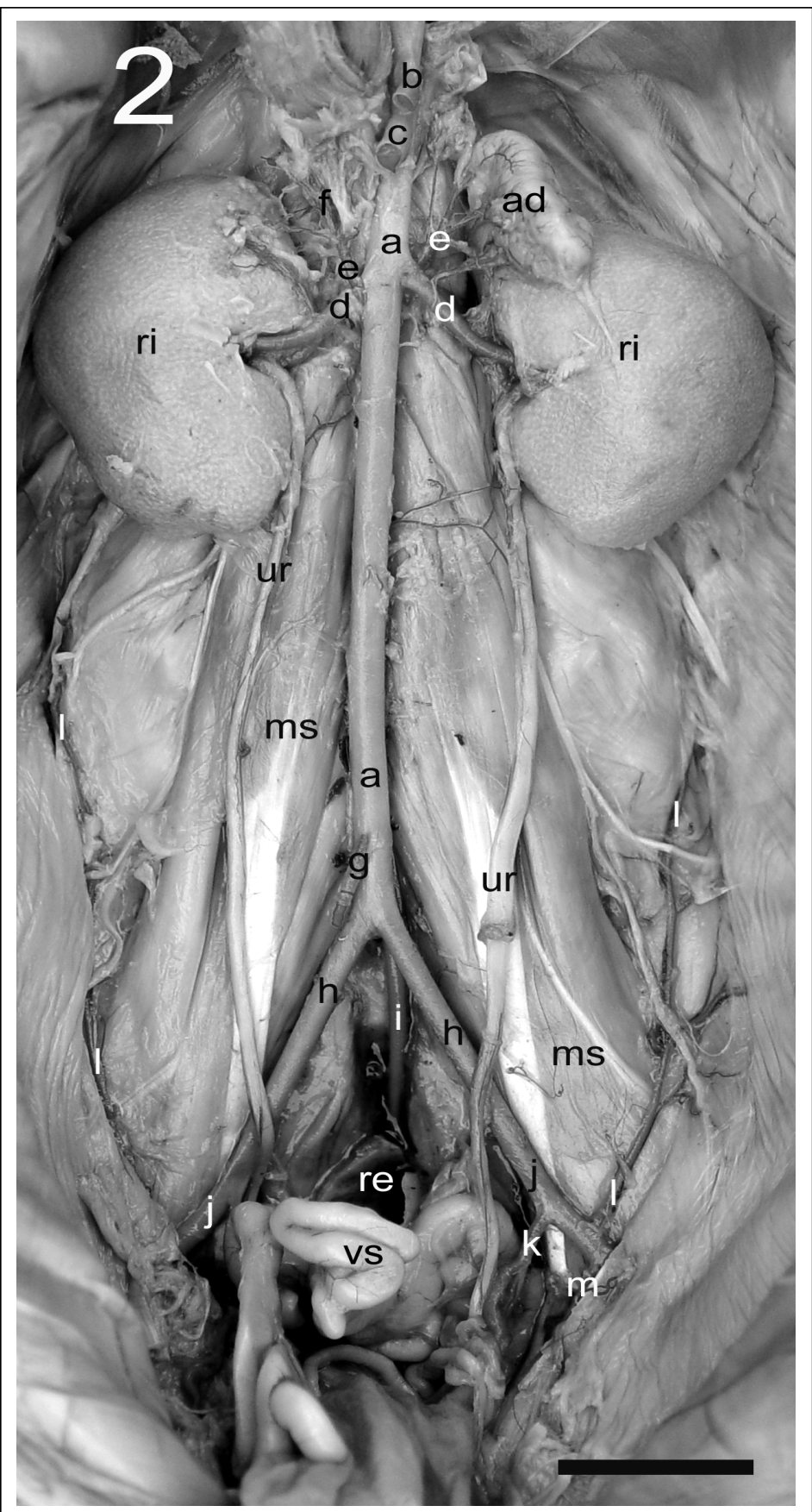

Figura 2 - Fotografia em vista ventral da parede abdominal dorsal, retirado o trato digestivo, para salientar a aorta abdominal e seus ramos colaterais e terminais: a - aorta abdominal; b - a. celíaca; c - a. mesentérica cranial; d - a. renal; e - a. frênico-abdominal; f - a. frênica caudal; g -a. mesentérica caudal; h - a. ilíaca comum; i - a. sacral mediana; $\mathrm{j}$ - a. ilíaca externa; $\mathrm{k}$ - a. umbilical; $\mathrm{l}$ - a. circunflexa ilíaca profunda; m - tronco pudendo-epigástrico; ri - rim; ur - ureter; ms - musculatura sublombar; re - reto, vs - vesícula seminal. Barra $=20 \mathrm{~mm}$.

A artéria umbilical, nos machos (Figuras 1 e 2), originou ramos vesicais para a bexiga urinária e glândulas anexas do aparelho genital masculino, continuando-se como artéria testicular que saiu pelo anel inguinal, incorporada ao funículo espermático até atingir o testículo. A artéria umbilical, nas fêmeas, além de lançar ramos vesicais para a bexiga urinária, continuou-se cranialmente como artéria útero-ovárica irrigando todo o útero e os ovários.

A artéria ilíaca externa lançou latero-cranialmente uma artéria circunflexa ilíaca profunda (Figuras 1 e 2 ), que percorreu os dois terços caudais da parede dorsolateral da cavidade abdominal, anastomosando-se com a artéria abdominal cranial. A artéria circunflexa ilíaca profunda direita foi única em 96,7\% das amostras, sendo que em 24 casos ela foi emitida da artéria ilíaca externa, cranialmente ou na mesma altura da origem do tronco pudendoepigástrico. Já em 4 amostras, a artéria circunflexa ilíaca profunda originou-se da artéria ilíaca externa, caudalmente à origem do tronco pudendo-epigástrico, e, em um dos achados, a artéria circunflexa ilíaca profunda foi ramo colateral do tronco pudendoepigástrico, logo após sua origem. A artéria circunflexa ilíaca profunda direita foi dupla em 3,3\% das amostras, sendo que o primeiro vaso foi lançado cranialmente à origem do tronco pudendo-epigástrico.

A artéria circunflexa ilíaca profunda esquerda foi única em $80 \%$ das amostras, sendo que em 19 casos ela foi emitida da artéria ilíaca externa, cranialmente ou na mesma altura da origem do tronco pudendo-epigástrico. Já em 4 amostras, a artéria circunflexa ilíaca profunda originou-se da artéria ilíaca externa, caudalmente à origem do tronco pudendo-epigástrico, e, em um dos achados, a artéria circunflexa ilíaca profunda foi ramo colateral do tronco pudendo-epigástrico, logo após sua origem. A artéria circunflexa ilíaca profunda esquerda foi dupla em $20 \%$ das amostras. Em três casos, ambos os vasos originaram-se da artéria ilíaca externa sendo que o primeiro vaso foi cranial à origem do tronco pudendo-epigástrico e o segundo foi emitido na mesma altura deste. Já em duas amostras, o primeiro 
vaso foi lançado cranial à origem do tronco pudendoepigástrico e o segundo vaso caudal à origem deste. Ainda em uma peça o primeiro vaso foi ramo da artéria ilíaca externa, cranial à origem do tronco pudendoepigástrico e o segundo vaso foi ramo direto deste tronco.

A artéria ilíaca externa, pouco antes de mergulhar no anel femoral, emitiu caudo-medialmente o tronco pudendo-epigástrico (Figura 1). O tronco pudendo-epigástrico apresentou-se curto em relação à origem da artéria epigástrica caudal em 53,4\% das peças à direita e $50 \%$ à esquerda. Já em $23,3 \%$ dos achados à direita e $26,6 \%$ à esquerda, este tronco foi longo e em $20 \%$ à direita e $16,7 \%$ à esquerda este tronco foi de tamanho médio. Entretanto, em 3,3\% das amostras, à direita, e 6,7\% à esquerda, não houve a formação do tronco, pois a artéria epigástrica caudal era ramo da artéria ilíaca externa.

A artéria epigástrica caudal (Figura 1) projetou-se ventro-látero-cranialmente na parede abdominal, percorrendo a parede abdominal ventral, irrigando o músculo reto abdominal, anastomosandose em ósculo com os ramos da artéria epigástrica cranial proveniente da artéria torácica interna, cranialmente à altura da cicatriz umbilical. A artéria pudenda externa saiu pelo canal inguinal, indo irrigar a genitália externa. A artéria ilíaca externa abandonou a cavidade abdominal, através do anel femoral, como artéria femoral, indo irrigar o membro pélvico.

\section{DISCUSSÃO}

A aorta abdominal foi a parte da aorta descendente que penetrou na cavidade abdominal após atravessar o hiato aórtico do diafragma. No cão apresentou sete artérias lombares pares. Destas as duas ou as três primeiras surgiram da aorta torácica e as restantes da aorta abdominal. As sétimas artérias lombares surgiram de um tronco comum, quer da parte terminal da aorta abdominal, quer da artéria sacral mediana ou da artéria ilíaca interna (GHOSHAL, 1986; EVANS, 1993). A nutria apresentou de 6 a 8 artérias lombares, porém ímpares, originadas da superfície dorsal da aorta abdominal, sendo que a última artéria lombar originou-se quase sempre da artéria sacral mediana.

A artéria frênico-abdominal par surgiu da aorta abdominal entre a artéria mesentérica cranial e a artéria renal. A artéria frênico-abdominal, à esquerda, foi bastante constante, enquanto que a artéria direita surgiu em alguns casos da artéria renal direita (GHOSHAL, 1986; EVANS, 1993). Na nutria, a artéria frênico-abdominal foi normalmente emitida como ramo colateral das artérias renais. A artéria frênico-abdominal foi um tronco comum para as artérias frênica caudal e abdominal cranial. A artéria frênica caudal e a artéria abdominal cranial também se originaram separadamente. Sua terminação caudal normalmente anastomosou-se com as terminações da artéria circunflexa ilíaca profunda, como também foi observado no cão por GHOSHAL (1986) e EVANS (1993)

No cão a artéria aorta abdominal freqüentemente terminou-se em duas artérias ilíacas internas e sacral mediana. A artéria ilíaca externa surgiu da face lateral da aorta abdominal e foi considerada por EVANS (1993) como o maior ramo parietal da aorta abdominal. Segundo CARVALHO et al. (1990), a aorta abdominal no gambá terminou-se como artérias ilíacas comuns, as quais emitiram as artérias ilíacas interna e externa. Pelos desenhos esquemáticos de COOK (1965), a aorta abdominal no rato terminou em duas artérias ilíacas comuns que lançaram a artéria ilíaca interna, continuando-se como a artéria ilíaca externa, o que também foi observado na nutria, sendo que a artéria sacral mediana surgiu da face dorsal da aorta abdominal antes da divisão terminal. Para CULAU et al. (2002), em $68,2 \%$ dos gambás, a artéria sacral mediana originouse diretamente da bifurcação da aorta abdominal em artérias ilíacas comum direita e esquerda.

No gambá, a artéria circunflexa ilíaca profunda originou-se da artéria ilíaca comum, na altura de sua bifurcação em artérias ilíacas externa e interna em 54,5\% dos casos nos dois antímeros (CULAU et al., 2002). Na nútria a artéria circunflexa ilíaca profunda foi normalmente ramo da artéria ilíaca externa, próximo à origem do tronco pudendo-epigástrico.

No cão, segundo GHOSHAL (1986) e EVANS (1993), o tronco pudendo-epigástrico foi muito curto e próximo ao anel inguinal profundo emitiu a artéria epigástrica caudal (profunda) e seguiu através do canal inguinal como artéria pudenda externa. Às vezes o tronco pudendo-epigástrico esteve ausente e as artérias epigástrica caudal e pudenda externa originaram-se separadamente da artéria femoral profunda, que foi ramo da artéria ilíaca externa. Na nutria, o tronco pudendo-epigástrico apresentou-se curto em relação à origem da artéria epigástrica caudal na maioria dos casos. Além disso, em 3,3\% das preparações à direita e 6,7\% à esquerda, não houve a formação do tronco, pois a artéria epigástrica caudal foi ramo da artéria ilíaca externa. A artéria pudenda externa saiu pelo canal inguinal, irrigando a genitália externa.

\section{CONCLUSÃO}

Com base nos resultados obtidos pelo presente trabalho, concluímos que os ramos colaterais 
parietais diretos da aorta abdominal são: as artérias lombares ímpares e a artéria sacral mediana. Os ramos colaterais parietais indiretos são as artérias: frênicoabdominais, circunflexas ilíacas profundas e epigástricas caudais. Os ramos terminais são as artérias ilíacas comuns com seus ramos e as artérias ilíacas interna e externa.

\section{FONTES DE AQUISIÇÃO}

aHeparin - Cristália Produtos Químicos Farmacêuticos Ltda., Itapira, SP.

bTiopental - Cristália Produtos Químicos Farmacêuticos Ltda., Itapira, SP.

cCola 603 - Bertoncini Ltda, São Paulo, SP.

dSuvinil corante - BASF S.A., São Bernardo do Campo, SP.

\section{REFERÊNCIAS}

BAROFFIO, R.A. et al. Nuestra nutria. 2.ed. Buenos Aires: Hemisferio Sur, 1979. 159p.

CARVALHO, R.G., et al. About the arterial blood vessels pelvic opossum's (Didelphis albiventris) behaviour. Anat Anz, v.70, p.367-372, 1990.

COOK, M.J. The anatomy of the laboratory mouse. 2.ed. London: Academic, 1965. 143p.
CULAU, P.O.V. et al. Ramos terminais da artéria aorta abdominal no gambá (Didelphis albiventris). In: CONGRESSO BRASILEIRO DE ANATOMIA, 20., 2002, Maceió, AL. Anais... Maceió: Sociedade Brasileira de Anatomia, 2002. 183p. p.91.

EVANS, H.E. The heart and the arteries. In: EVANS, H.E. Miller's anatomy of the dog. 3.ed. Philadelphya: Saunders, 1993. Cap.11, p.586-681.

GHOSHAL, N.G. Coração e artérias do carnívoro. In: GETTY, R. Sisson/Grossman anatomia dos animais domésticos. 5.ed. Rio de Janeiro: Interamericana. 1986. V.2, cap.55, p.14971550 .

INTERNATIONAL COMMITTEE ON VETERINARY GROSS ANATOMICAL NOMENCLATURE. Nomina anatomica veterinaria. 5.ed. Knoxville, 2005. 166p.

MACHADO, G.V. et al. Suprimento arterial para as glândulas adrenais no ratão-do-banhado (Myocastor coypus Molina, 1782). Archieves of Veterinary Science, v.7, n.2, p.9-14, 2002.

MACHADO, G.V. et al. A artéria celíaca e seus ramos no ratãodo-banhado (Myocastor coypus - Rodentia: Mammalia). Biotemas, v.15, n.2, p.41-54, 2002.

SILVA, F. Mamíferos silvestres do Rio Grande do Sul. Porto Alegre: Fundação Zoobotânica do Rio Grande do Sul, 1994. 282p. 